\title{
Comparison of different image analysis algorithms on MRI to predict physico-chemical and sensory attributes of loin
}

Caballero, Daniel; Caro, Andrés; Dahl, Anders Bjorholm; Ersbøll, Bjarne Kjær; Amigo, José Manuel; Pérez-Palacios, Trinidad; Antequera, Teresa

\section{Published in:}

Chemometrics and Intelligent Laboratory Systems

Link to article, DOI:

10.1016/j.chemolab.2018.04.008

Publication date:

2018

Document Version

Peer reviewed version

Link back to DTU Orbit

Citation (APA):

Caballero, D., Caro, A., Dahl, A. B., Ersbøll, B. K., Amigo, J. M., Pérez-Palacios, T., \& Antequera, T. (2018). Comparison of different image analysis algorithms on MRI to predict physico-chemical and sensory attributes of loin. Chemometrics and Intelligent Laboratory Systems, 180, 54-63.

https://doi.org/10.1016/j.chemolab.2018.04.008

\section{General rights}

Copyright and moral rights for the publications made accessible in the public portal are retained by the authors and/or other copyright owners and it is a condition of accessing publications that users recognise and abide by the legal requirements associated with these rights.

- Users may download and print one copy of any publication from the public portal for the purpose of private study or research.

- You may not further distribute the material or use it for any profit-making activity or commercial gain

- You may freely distribute the URL identifying the publication in the public portal 


\section{Accepted Manuscript}

Comparison of different image analysis algorithms on MRI to predict physico-chemical

and sensory attributes of loin

Daniel Caballero, Andrés Caro, Anders B. Dahl, Bjarne K. ErsbØII, José Manuel Amigo, Trinidad Pérez-Palacios, Teresa Antequera

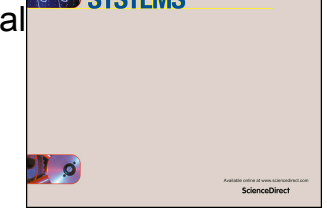

PII:

S0169-7439(17)30755-4

DOI:

10.1016/j.chemolab.2018.04.008

Reference: CHEMOM 3618

To appear in: Chemometrics and Intelligent Laboratory Systems

Received Date: 27 November 2017

Revised Date: 16 March 2018

Accepted Date: 6 April 2018

Please cite this article as: D. Caballero, André. Caro, A.B. Dahl, B.K. ErsbØII, José.Manuel. Amigo, T. Pérez-Palacios, T. Antequera, Comparison of different image analysis algorithms on MRI to predict physico-chemical and sensory attributes of loin, Chemometrics and Intelligent Laboratory Systems (2018), doi: 10.1016/j.chemolab.2018.04.008.

This is a PDF file of an unedited manuscript that has been accepted for publication. As a service to our customers we are providing this early version of the manuscript. The manuscript will undergo copyediting, typesetting, and review of the resulting proof before it is published in its final form. Please note that during the production process errors may be discovered which could affect the content, and all legal disclaimers that apply to the journal pertain. 


\section{TITLE}

Comparison of different image analysis algorithms on MRI to predict physicochemical and sensory attributes of loin.

\section{AUTHORS}

Daniel Caballero a*, Andrés Caro a, Anders B. Dahl b, Bjarne K. Ersbøll c, José Manuel Amigo d, Trinidad Pérez-Palacios e, and Teresa Antequera ${ }^{e}$

a Computer Science Department, Research Institute of Meat and Meat Product (IproCar), University of Extremadura, Av/ Universidad S/N, ES-10003 Cáceres, Spain.

b Department of Applied Mathematics and Computer Science, Technical University of Denmark, Richard Petersen Plads, Building 324, DK-2800, Kongens Lyngby, Denmark.

c Department of Informatics and Mathematical Modeling, Technical University of Denmark, Richard Petersen Plads, Building 324, DK-2800, Kongens Lyngby, Denmark.

d Department of Food Science, Quality and Technology, Faculty of Life Science, University of Copenhagen, Rolighedsvej 30, DK-1958, Frediksberg C, Denmark.

e Food Technology Department, Research Institute of Meat and Meat Product (IproCar), University of Extremadura, Av/ Universidad S/N, ES-10003 Cáceres, Spain.

* Corresponding author: Tel: +34 927 257123. Fax: +34 927 257110. E-mail: dcaballero@unex.es 
Author's e-mails: dcaballero@unex.es, andresc@unex.es, abda@dtu.dk, bker@dtu.dk, imar@food.ku.dk, triny@unex.es and tantero@unex.es, respectively. 


\section{ABSTRACT}

Computer vision algorithms on MRI have been presented as an alternative to destructive methods to determine the quality traits of meat products. Since, MRI is non-destructive, non-ionizing and innocuous methods. The use of fractals to analyze MRI could be another possibility for this purpose. In this paper, a new fractal algorithm is developed, to obtain features from MRI based on fractal characteristics. This algorithm is called OPFTA (One Point Fractal Texture Algorithm). Three fractal algorithms (Classical Fractal Algorithm -CFA-, Fractal Texture Algorithm -FTA- and OPFTA) and three classical texture algorithms (Grey level co-occurrence matrix -GLCM-, Grey level run length matrix -GLRLM- and Neighbouring grey level dependence matrix -NGLDM-) were tested in this study. The results obtained by means of these computer vision algorithms were correlated to the results obtained by means of physico-chemical and sensory analysis. CFA reached low relationship for the quality parameters of loins, the remaining algorithms achieved correlation coefficients higher than 0.5 noting OPFTA that reached the highest correlation coefficients in all cases except for the L* coordinate color that GLCM obtained the highest correlation coefficient. These high correlation coefficients confirm the new algorithm as an alternative to the other computer vision approaches in order to compute the physicochemical and sensory parameters of meat products in a non-destructive and efficient way.

\section{KEYWORDS}

Fractal, texture features, algorithms, data mining, food technology

\section{ABBREVIATIONS}


MRI: Magnetic Resonance Imaging. PCA: Principal Component Analysis. KDD: Knowledge Discovery in Databases. MLR: Multiple Linear Regression. SE: Spin Echo. FOV: Field-of-view. TE: Echo time. TR: Repetition time. CFA: Classical fractal algorithm. FTA: Fractal texture algorithm. GLCM: Grey level cooccurrence matrix. GLRLM: Grey level run length method. NGLDM: Neighboring grey level dependence matrix. OPFTA: One point of fractal texture algorithm. ROI: Region of interest. ENE: Energy. ENT: entropy. COR: Correlation. HC: Haralick's correlation. IDM: Inverse difference moment. INE: Inertia. CS: Cluster Shade. CP: Cluster Prominence. CON: Contrast. DIS: Dissimilarity. SRE: Short run emphasis. LRE: Large run emphasis. GLNU: Gray level non-uniformity. RLNU: Run length non-uniformity. RPC: Run percentage. LGRE: Low gray-level run emphasis. HGRE: High gray-level run emphasis. SRLGE: Short run low gray-level emphasis. LRLGE: Large run gray-level emphasis. SRHGE: Short run high graylevel emphasis. LRHGE: Large run high gray-level emphasis. SNE: Small number emphasis. LNE: Large number emphasis. NNU: Number non-uniformity. SM: Second moment. UNI: Uniformity. EMP: Emphasis. JC: Jorna's correlation. HOM: Homogeneity. EFI: Efficiency. WEKA: Waikato environment for knowledge analysis. R: Correlation coefficient. MAE: Mean absolute error. 


\section{INTRODUCTION}

Magnetic Resonance Imaging (MRI) is a non-destructive, non-invasive, nonintrusive, non-ionizing and innocuous technique to acquire images. This makes MRI an alternative for determining quality and sensory parameters of meat and meat products. Several works have been carried out to determine quality parameters of dry-cured products by MRI, allowing to monitor the ripening process of Iberian [1], Parma [2] and San Daniele [3] hams. Iberian hams were classified as a function of pig feeding background [4 - 5] and some physicochemical and sensory traits were predicted [6 - 7]. Other authors have characterized Slovenian dry-cured hams [8]. On loins, an adequate product classification as a function of pig breeding and the classification of the product in three quality degrees (low, medium and high) [9 - 10] and some physicochemical and sensory parameters have been predicted [1 1 - 13].

The extraction of textural information from images is very common to explore parameters related to food quality. Antequera et al. [14] applied computational texture algorithms as a tool to predict intramuscular fat and sensory characteristics. Ávila et al. [15] analyzed marbling and fat level in Iberian loin based on texture features. Texture algorithms were applied to study the marbling on beef [16], to predict the tenderness of cooked beef from images of fresh beef [17], or to classify bovine meat [18]. The efficiency of the computational texture algorithms to solve problems related to meat product, in fresh and dry-cured products, have been proved [19 - 20]. Recently, Caballero applied texture features to monitor the diffusion of salt in Iberian ham [21]. All these methods integrated matrices based on second-order statistics $[4,5,14]$, that relate computational texture features and, some physico-chemical and 
sensory attributes were found. Additionally, using first-order statistics, moisture, fat and salt contents were monitored with different processing methods [22].

The fractal concept studies the degree of symmetry or self-similarity found in a structure at all scales. Mainly, the use of fractals allows the identification of recurring patterns, removing the possibility of image compression [23-26]. In recent years, there is a growing interest in the use of fractal analysis techniques. Mainly, this technique has been applied to characterize the microstructure of different types of food [27 - 30], microstructure of beef [31] and poultry meat [32]. In relation to the use of fractals to predict quality traits of food, Tsuta et al. [33] applied them to predict the sugar content of melons and Polder et al. [34] measured the chlorophyll of tomato by applying of fractals. However, to the best of our knowledge, the use of fractal analysis to predict quality parameters on meat products only it has been applied in one study [12].

Regarding the data analysis, usual statistical tools such as Pearson's correlation coefficients or Principal Components Analysis (PCA) have been normally applied $[4,10,14]$. New advances in information technologies have made it possible to collect, store and process massive large and complex datasets [35]. This is known as Knowledge Discovery in Databases (KDD). Data mining is one of the stage in the KDD process [36], as a non-trivial process of finding knowledge and potentially useful information from data stored in repositories [37]. There are several data mining algorithms when modelling continuous data. Multiple Linear Regression (MLR) is one of the classical approaches. It models the linear relationship between a dependent variable (target) and one or more independent variables (predictors). MLR was introduced in 1974 [38] and popularized in 1986 [39]. In food technology, MLR was applied to model wine 
preferences [40], to predict quality traits in beef [41] and lamb [42]. Recently, Pérez-Palacios et al. [6, 13] and Caballero et al. $[7,11,12,21]$ have applied MLR into Iberian meat products.

This paper is organized as follows: Section 2 presents the Materials and Methods used in this study. Section 3 describe the obtained results and their discussion. Section 4 draws the main conclusions and their implications.

This paper aims at 1) developing an algorithm for studying texture features based on fractal and second-order statistics and 2) comparing different image analysis algorithms and its application on MRI images in order to predict quality parameters of loins.

\section{MATERIAL AND METHODS}

The prediction of quality and sensory attributes on this study was carried out with data from 5220 MRI images from Iberian loins.

\subsection{IMAGE ACQUISITION}

MRI images were generated at the "Animal Source Foodstuffs Innovation Service" (SiPA) of the Faculty of Veterinary Science at University of Extremadura (Cáceres, Spain) A low-field MRI scanner (ESAOTE VET-MR E-SCAN XQ 0.18 T) with a hand/wrist coil used. Spin Echo (SE) T1-weighted sequence was applied. In $\mathrm{SE}$, a $90^{\circ}$ radiofrequency excitation pulse is followed by a $180^{\circ}$ radiofrequency pulse to eliminate static magnetic field dissimilarity. The following parameters were used: field-of-view (FOV): 150x150 mm²; slice thickness: $4 \mathrm{~mm}$; flip angle: 90; matrix size: 256 × 204; phase encode: 204; number of acquisition: five per sample with nine different configurations on 
echo time (TE) and repetition time (TR). Twenty nine slices per loin were obtained and the MRI acquisition took 50 min for each loin.

The MRI acquisition was performed at $23^{\circ} \mathrm{C}$. All images were acquired in DICOM format with $512 \times 512$ resolution and 256 gray levels.

\subsection{IMAGE ANALYSIS ALGORITHMS}

Once the MRls of loins were obtained, six image analysis algorithms were applied to extract numerical data from them. The classical fractal algorithm (CFA) and Fractal Texture Algorithm (FTA) are applied directly on the images. However, the four remaining algorithms, gray level co-occurrence matrix (GLCM), gray level run length matrix (GLRLM), neighbouring gray level dependence matrix (NGLDM) and one point fractal texture algorithm (OPFTA) require a previous step, this step is the selection of largest area rectangle inscribed on the image closed contour [43]. These rectangles are called Region of Interest (ROI). These ROls are rectangle because is the geometry figure that best fitting to our algorithms, and, later, the algorithms are applied on these ROIs [44].

\subsubsection{GRAY LEVEL CO-OCCURRENCE MATRIX}

GLCM [45 - 46] was computed by counting the number of times that each pair of gray levels occurred at a given distance $d$ in all directions. In this matrix, each item $p(i, j)$ denotes the number of times that two neighbouring pixels separated by distance $d(d=1$ in this case) occur on the image, one with gray level $i$ and the other with gray level $j$, in all $2 \mathrm{D}$ directions: $0^{\circ}, 45^{\circ}, 90^{\circ}, 135^{\circ}, 180^{\circ}$, $225^{\circ}, 270^{\circ}$ and $315^{\circ}$. Ten computational texture features were obtained from this

method proposed by Haralick [45]: energy (ENE), entropy (ENT), correlation 
(COR), Haralick's correlation (HC), inverse difference moment (IDM), inertia (INE), cluster shade (CS), cluster prominence (CP), contrast (CON) and dissimilarity (DIS). Table 1 shows the equations that allow computing these features.

\subsubsection{GRAY LEVEL RUN LENGTH MATRIX}

GLRLM [47 - 48] includes runs into the image, i.e., a set of consecutive pixels in the image with the same gray level value. A large number of neighbouring pixels of the same gray level represents a coarse texture, a small number of these pixels represents a fine texture, and the lengths of the texture primitives in different directions can serve as texture description [49]. The runs with the same gray level were computed in four different directions: $0^{\circ}, 45^{\circ}, 90^{\circ}$ and $135^{\circ}$. Eleven computational texture features were obtained from this method [47]: Short run emphasis (SRE), Long run emphasis (LRE), gray level non-uniformity (GLNU), run length non-uniformity (RLNU), Run percentage (RPC), Low graylevel run emphasis (LGRE), high gray-level run emphasis (HGRE), Short run low gray-level emphasis (SRLGE), Long run low gray-level emphasis (LRLGE), short run high gray-level emphasis (SRHGE) and long run high gray-level emphasis (LRHGE). Table 2 shows the equations that allow computing these features.

\subsubsection{NEIGHBOURING GRAY LEVEL DEPENDENCE MATRIX}

NGLDM uses angular independent features by considering the relationship between an element and all its neighbouring elements at one time rather than one direction at a time [50-51]. In this method, the neighbouring are square and the dimension of these square are $3 \times 3$ and the distance $d(d=1)$ between neighbouring pixels. This process eliminates the angular dependency while simultaneously reducing the calculations required to process an image. It is 
based on the assumption that the gray-level spatial dependence matrix of an image can adequately specify this texture information. Five computational texture features were obtained using this method [51]: small number emphasis (SNE), large number emphasis (LNE), number non-uniformity (NNU), second moment (SM) and entropy (ENT). Table 3 shows the equations that allow computing these features.

\subsubsection{CLASSICAL FRACTAL ALGORITHM}

CFA [24] studies the pattern of repetition in the image. The method measures the number of boxes (small fractions of the image depending on the size of the original image) needed to cover an area occupied by the object as a function of the size of boxes. This is calculated by computing the so-called local exponent with different box sizes. The local exponent, $D$, is the variation of the number of objects $(N)$ depending on the box size $(R)$.

$D=-\frac{\Delta \ln N}{\Delta \ln R}$

The fractal dimension is the value of the number of $D$ when it remains constant respect to the box size. Quantification of the fractal dimension of each simple image was calculated using the compression box counting package (toolbox downloaded

from

http://www.mathworks.com/matlabcentral/fileexchange/13063-boxcount - last accessed June 2017, for MATLAB (The Mathworks Inc., Natick, Massachusetts, U.S.A.)). Nine fractal dimensions were computed in this algorithm: BOX1, BOX2, BOX3, BOX4, BOX5, BOX6, BOX7, BOX8 and BOX9.

\subsubsection{FRACTAL TEXTURE ALGORITHM}


FTA [52] is a novelty texture algorithm based on fractal characteristics that are obtained from a two dimensional variation of Minkowski-Bouligand algorithm [24]. The fractal features reflect the number of times that a pattern is repeated in each image depending of box size calculated in each case. The fractal characteristics were gathered to create a vector. Each vector was computed by based on second order statistics, obtaining ten computational texture features on each one: Uniformity (UNI), Entropy (ENT), Correlation (COR), Inverse Difference Moment (IDM), Inertia (INE), Contrast (CON), Emphasis (EMP), Jorna's Correlation (JC), Cluster shade (CS) and Cluster Prominence (CP) [53 - 54]. Table 4 shows the equations that allow computing these features.

\subsubsection{ONE POINT FRACTAL TEXTURE ALGORITHM}

OPFTA is the algorithm introduced in [55] and proposed in this paper, OPFTA is a novelty algorithm based on features obtained from fractal values. Figure 1 summarizes the flow chart of this algorithm.

First, the image acquisition process obtained sets of MRI, in a high resolution (pixel resolution $0.23 \times 0.23 \mathrm{~mm}$ ) (Figure $1 \mathrm{~A}$ ). When the images were acquired, the largest area rectangle inscribed in the contour of the loin was selected (Figure 1B) [43]. Then, each rectangle was divided into smaller rectangles of 32 x 32 pixels, so called ROI (Figure 1C). At this point, a two dimensional variation of the Minkowski-Bouligand algorithm [24] was applied on each one of the ROI in order to obtain local exponents with the different box sizes (powers of 2). Algorithm 1 was used to compute the local exponents for OPFTA. These local exponents reflect the number of times that a pattern is repeated for each ROI depending of the size of the boxes that they have been calculated in each case. From all local exponent, we select the local exponent with the box size 
equal to eight (Figure 1D), this value is the most representative. After that, we gathered one value for each $\mathrm{ROI}$ in order to create a matrix with the fractal values. Each cell of the matrix represents one ROI from the image (Figure 1E).

Finally, seven features were computed on each matrix (Figure 1F). These features were calculated based on second order statistics [53 - 54]. The features calculated were the following: Uniformity (UNI), entropy (ENT), correlation (COR), homogeneity (HOM), inertia (INE), contrast (CON) and efficiency (EFI). UNI indicates the uniformity of the gray level, i.e. there is a large clusters of pixels with the same or similar gray levels. ENT describe the texture of the images, concretely, if the image has got messy o has not got a messy texture. COR indicates the relationship between the gray levels and the energy of the image. HOM describe the homogeneity of the images. INE reflect that the clusters of the pixels with the same or similar levels of gray are small. CON describe the symmetry of the structure of the images. EFI indicates the relationship between the gray levels and the size of the clusters of the pixel with the same or similar gray levels [49]. Table 5 shows the equations to calculate each feature from the values of the previously computed matrix.

Algorithm 2 shows how the features are computed from the images.

The proposed algorithm OPFTA supposes a great novelty in obtaining texture features on MRI images of food (loins in this study), since the obtained data are not based on texture features, since each cell of the matrix represents a fractal value and the texture is based on the fractal values.

\subsection{PHYSICO-CHEMICAL DATASET}


One dataset was constructed from data obtained from physico-chemical analysis on Iberian loins. These analyses were carried out by means of traditional methods in order to obtain values for:

- The moisture was determined at $100 \pm 2{ }^{\circ} \mathrm{C}$ by the official method $[56$ reference 935.29].

- The lipid content of loins was determined gravimetrically with chloroform:methanol $(2: 1, v / v)$, according to the method described in Pérez-Palacios et al. [57].

- The water activity, the system Lab Master-aw (NOVASINA AG, Switzerland) was used after calibration at $20-22^{\circ} \mathrm{C}$.

- Instrumental color was measured using a Minolta CR-300 colorimeter (Minolta Camera Corp., Meter Division. Ramsey, NJ) with illuminant D65, a $0^{\circ}$ standard observer and a $2.5 \mathrm{~cm}$ port/viewing area. The following color coordinates were determined: lightness $\left(L^{*}\right)$, redness $\left(\mathrm{a}^{*}\right)$ and yellowness (b*). The colorimeter was standardized before use with $\mathrm{a}$ white tile having the following values: $L^{*}=93.5, a^{*}=1.0$ and $b^{*}=0.8$.

- Salt content was determined volumetrically in dry-cured loins by the official method [56 reference 971.19].

\subsection{SENSORY DATASET}

One dataset was constructed from data obtained from sensory analysis on Iberian loins. These analyses were assessed by a trained panel of thirteen members, they were developed in tasting rooms with the conditions specified in UNE-EN-ISO 8589:2010 regulation. All sessions were conducted at room temperature $\left(22^{\circ} \mathrm{C}\right)$ in a sensory room equipped with white fluorescent lighting $(220-230 \mathrm{~V}, 35 \mathrm{~W})$. The software used to record scores in the sensory sessions 
was FIZZ Network (version 2.2, Biosystems, France). For each loin, two slices of 1.5 $\mathrm{mm}$ were given to the panelists. Slices were obtained using a commercial slicing machine and were served to the panelists on plates. The panel sessions were held mid-morning, approximately $4 \mathrm{~h}$ after breakfast. Three samples randomly presented to the panelists were analyzed in each session. Approximately $200 \mathrm{~mL}$ water was provided to the panelists [58].

Eleven sensory traits of Iberian loin were analyzed [14]: redness of lean, brightness of lean, marbling, odour intensity, hardness, juiciness, salty taste, flavor intensity, cured flavor, rancid flavor and flavor persistence. These traits were assessed on a non-structured scale of $0-10$, the panel average was recorded for each sample.

\subsection{DATABASES}

Two databases were constructed to carried out this study. One of them were constructed with the values of the features from the application of image analysis algorithms on MRI of loins and the dataset of the physico-chemical data of the same loins. The other one were constructed with the values of the data of dataset of the sensory data of loins and the values of the features from the image analysis algorithms on MRI of the same loins.

\subsection{PREDICTIVE TECHNIQUES}

The free software WEKA (Waikato Environment for Knowledge Analysis) (http://cs.waikato.ac.nz/ml/weka - last accessed: June 2017) was used for carrying out the predictive techniques of data mining.

MLR as predictive technique of data mining was applied on the databases. Predictive techniques allow creating future models that can be predicted from 
current data by trend analysis [59 - 60]. MLR is used to represent linear relationship between a dependent variable and several independent variables. This technique obtains a linear regression equation, which can be used to predict future values [35].

$y=\omega_{0}+\sum_{1}^{n} \omega_{i} x_{i}$

The M5 method of attribute selection was applied in our experiments. This method steps through the attributes removing the one with the smallest standardized coefficient until no improvement is observed in the estimation of the error. A ridge value of $1.0 \times 10^{-4}$ was applied too. The estimation procedure applied was 10-fold cross validation [61], where the data is divided into 10 partitions of equal size. One subset is tested each time and the remaining data are used for fitting the model. The process is repeated sequentially until all subset have been tested. Therefore, all data are used for training and testing. However, although this method requires 10 repetition analysis, this is a robust method.

The correlation coefficient (R) was used for evaluating the goodness of the prediction according to rules given by Colton [62]. This author considered that when correlation coefficients varies from 0 to 0.25 , then there was a little or no relationship; from 0.25 to 0.50 , fair degrees of relationship; from 0.50 to 0.75 , moderate to good relationship; and from 0.75 to 1 , very good to excellent relationship.

$R=\sqrt{\frac{\sum_{i=1}^{n}\left(f_{i}-\bar{y}\right)^{2}}{\sum_{i=1}^{n}\left(y_{i}-\bar{y}\right)^{2}}}$

Where $\mathrm{fi}$ is the predicted value, $\mathrm{yi}$ is the real value and is the average one. 
In addition to this, Mean Absolute Error (MAE) of prediction [63] was used to evaluate the prediction results too. The MAE measures the difference between real values and predicted ones. Values of MAE lower than 2 are appropriate [64-65]. It is given by the following equation:

$M A E=\frac{1}{n} \sum_{i=1}^{n}\left|f_{i}-y_{i}\right|$

Where $\mathrm{fi}$ is the predicted value and $\mathrm{yi}$ is the real one.

\section{RESULTS AND DISCUSSION}

In order to validate the OPFTA algorithm, this algorithm have been applied on a set of MRIs of loins and these values have been correlated with the values that determine the quality of this meat product. To evaluate the goodness of this new algorithm, five image analysis algorithms have been compared with the OPFTA algorithm.

Table 6 shows the computational complexity of all texture and fractal-based algorithms. OPFTA performs a computational complexity lower than classical texture and fractal algorithms. This low computational complexity from an industrial point of view is necessary in order to obtain a good performance on the extraction of features from the images. The fact of that our novelty algorithm, OPFTA, has got the same computational complexity that the classical GLCM texture algorithm proposed by Haralick [45] is a good indicator about the good performance of our algorithm.

The predicted values based on the six image analysis algorithms were correlated to the real values obtained by physico-chemical and sensory analysis. Thus, the $R$ values of equations were calculated (Table 7 and Table 8) and these values were used to evaluate the accuracy in the predictions. These 
results were analyzed taking into account the rules given by Colton [62]. This author considered that when correlation coefficients varies from 0 to 0.25 , then there was a little or no relationship; from 0.25 to 0.50 , fair degrees of relationship; from 0.50 to 0.75 , moderate to good relationship; and from 0.75 to 1 , very good to excellent relationship. As can be seen in the Table 7, for the physicochemical attributes of loins, CFA reached a fair degree of relationship for the most of characteristics ( $R<0.500$ ). The remaining algorithms, GLCM, GLRLM, NGLDM, FTA and OPFTA achieved relationship from moderate to excellent. Noting that the best correlation coefficients were obtained applying OPFTA as image analysis algorithm $(R>0.750)$, only for the $L^{*}$ coordinate of the instrumental colour, GLCM achieved higher correlation coefficient than the OPFTA. Table 8 shows the $\mathrm{R}$ values for sensory attributes of loins, the obtained results are similar than the results obtained for physico-chemical traits, CFA achieved a fair degree of relationship for all sensory attributes. For the remaining algorithms, the best correlation coefficients for all attributes were reached using OPFTA as image analysis algorithm ( $>0.750)$ followed by FTA, GLCM, NGLDM and GLRLM that showed from fair to very good relationship.

These facts joined to the good capability to simulate texture of the OPFTA algorithm, could validate the use of OPFTA as image analysis algorithm in order to predict physico-chemical and sensory attributes of loins. Since, the R values are slightly higher for OPFTA algorithm than the GLCM and FTA algorithms, noting that the most of attributes achieved $R$ values higher than 0.75 for these three algorithms (Table 7 and 8). In addition to this, OPFTA algorithm only computed seven computational seven features while GLCM and FTA computed ten features. 
Table 9 shows the prediction equations of the physico-chemical and sensory attributes of loins as a function of computational features from OPFTA algorithm. Comparing these equations with the prediction equations obtained applying the classical computational texture algorithms for the physicochemical [13] and sensory [11] attributes. The equations using OPFTA as image analysis algorithms only computed between three and seven features and the prediction equations from the classical texture algorithms require between seventeen and twenty-four features to predict the physico-chemical and sensory attributes of loins. Therefore, the prediction model based on features from OPFTA algorithm is simpler and easier to apply than the models developed using the classical texture feature algorithms, GLCM, NGLDM and GLRLM.

Taking into account the MAE between real lobtained from physico-chemical and sensory analysis) and predicted (obtained using the prediction equations) values (Table 9), for all attributes, physico-chemical and sensory, the MAE were lower than 2. Concretely, the values of MAE are between 0.014 and 1.822 . These values are considered appropriate in order to obtain predictions with a high degree of accuracy [64 - 65].

Taking into account the computation time, OPFTA is slightly faster than the GLCM and FTA algorithms (22 ms for OPFTA vs $27 \mathrm{~ms}$ for FTA and $46 \mathrm{~ms}$ for GLCM, computed using a usual laptop, INTEL i7-4510U, $2.6 \mathrm{GHz}, 16$ GB RAM). In addition to this, the use of classical computational texture algorithms have been validated to analyze MRI images from meat products in several studies [1, $6,7,10,11,13,15,21,49,67]$. 
Therefore, taking into account all these facts, the use of OPFTA as computer vision algorithm could be indicated in order to analyze MRI with the objective of to predict physico-chemical and sensory attributes of loins.

\section{CONCLUSIONS}

In this study, a novelty texture algorithm based on fractals and second order statistics has been proposed and validated. This algorithm, OPFTA, has been compared with other six image analysis algorithms, and its applicability has been showed in a real application, to predict physico-chemical and sensory attributes of loins.

In addition to this, the high values of the correlation coefficients shows the goodness and the suitability to use OPFTA with a high degree of accuracy.

Therefore, the use of OPFTA as image analysis algorithm join to MRI and predictive techniques could be a suitable tool for the meat industries in order to characterize their products in a non-destructive way.

\section{ACKNOWLEDGMENTS}

Daniel Caballero thanks the COST association, Farm Animal Imaging action (FAIM) (COST-FA1 102) and the European Social fund for the grant for a short stay (COST-STSM-FA1102-26642). The authors wish to acknowledge the funding received from the FEDER-MICCIN Infrastructure Research Project (UNEX-10-1E402) and Junta de Extremadura economic support for research group (GRU15173 and GRU15113). We also to thanks the "Montesano" company from Jerez de los Caballeros (Badajoz, Spain), the Animal Source Foodstuffs Innovation Service (SiPA) (Cáceres, Spain) from the University of Extremadura. The authors also wish to thanks to Dr. Francesco Savorani from the Polytechnic 
University of Turin (Turin, Italy), Dr. Knut Conradsen and Dr. Thomas Martini Jørgensen from the Technical University of Denmark (Kongens Lyngby, Denmark) for their direct contribution and support.

\section{REFERENCES}

[1] T. Antequera, A. Caro, P.G. Rodríguez, and T. Pérez-Palacios. Monitoring the ripening process of Iberian ham by Computer vision on magnetic resonance imaging. Meat Science, 76 (2007) 561-567.

[2] P. Fantazzini, M. Gombia, P. Schembri, N. Simoncini, and R. Virgili. Use of magnetic resonance imaging for monitoring Parma dry-cured ham processing. Meat science, 82 (2009) 219-227.

[3] L. Manzoco, M. Anese, S. Marzona, N. Innocente, C. Lagazio, and M.C. Nicoli. Monitoring dry-curing of $S$. Daniele ham by magnetic resonance imaging. Food Chemistry, 141 (2013) 2246-2252.

[4] T. Pérez-Palacios, T. Antequera, M.L. Durán, A. Caro, P.G. Rodríguez, and J. Ruiz. MRI-based analysis, lipid composition and sensory traits for studying Iberian dry-cured hams from pigs fed with different diets. Food Chemistry, 126 (2010) $1366-1372$.

[5] T. Pérez-Palacios, T. Antequera, M.L. Durán, A. Caro, P.G. Rodríguez, and R. Palacios. MRI-based analysis of feeding background effect on fresh Iberian ham. Food Research International, 43 (2011) 248-254.

[6] T. Pérez-Palacios, D. Caballero, A. Caro, P.G. Rodríguez, and T. Antequera. Applying data mining and Computer vision techniques to MRI to estimate quality traits in Iberian hams. Journal of food engineering, 131 (2014) 82-88. 
[7] D. Caballero, T. Antequera, A. Caro, M.L. Durán, and T. Pérez-Palacios. Data mining on MRI-Computational Texture Features to Predict Sensory Characteristics in Ham. Food and Bioprocess Technology, 9(4) (2016) 699-708.

[8] F. Bajd, M. Skrlep, M. Candek-Potokar, J. Vidmar, and I. Sersa. Application of quantitative magnetization transfer magnetic resonance imaging for characterization of dry-cured hams. Meat Science, 122 (2016) 109-118.

[9] E. Cernadas, T. Antequera, P.G. Rodríguez, M.L. Durán, R. Gallardo, and D. Villa. Magnetic resonance imaging to classify loin from Iberian pig. In G.A. Webb, P.S. Belton, A.M. Gil, and I. Delgadillo. (Eds.). Magnetic Resonance imaging in food science. A view to the future. The royal society of Chemistry. Cambridge, United Kingdom. 2001. pp. 239-245.

[10] E. Cernadas, P. Carrión, P.G. Rodríguez, E. Muriel, and T. Antequera. Analyzing magnetic resonance images of Iberian pork loin to predict its sensorial characteristics. Computer Vision and Image Understanding, 98 (2005) 345-361.

[11] D. Caballero, T. Antequera, A. Caro, M.M. Ávila, P.G. Rodríguez, and T. Pérez-Palacios. Non-destructive analysis of sensory traits of dry-cured loins by MRI-computer vision techniques and data mining. Journal of the food science and agriculture, 97 (2017) 2942-2952.

[12] D. Caballero, T. Pérez-Palacios, A. Caro, J.M. Amigo, A.B. Dahl, B.K. Ersbøll, and T. Antequera. Prediction of pork quality parameters by applying fractals and data mining on MRI. Food Research International, 99 (2017) 739-747.

[13] T. Pérez-Palacios, D. Caballero, T. Antequera, M.L. Durán, M.M. Ávila, and A. Caro. Optimization of MRI acquisition and texture analysis to predict physico- 
chemical parameters of loins by data mining. Food and bioprocess technology, 10 (2017) 750-758.

[14] T. Antequera, E. Muriel, P.G. Rodríguez, E. Cernadas, and J. Ruiz. Magnetic resonance imaging as a predictive tool for sensory characteristics and intramuscular fat content of dry-cured loin. Journal of the science of food and agriculture, 83 (2003) 268-274.

[15] M.M. Ávila, M.L. Durán, T. Antequera, R. Palacios, and M. Luquero. 3D reconstruction on MRI to analyze marbling and fat level in Iberian loin. In III Iberian Conference on Pattern Recognition and Image Analysis (IbPRIA), Girona, Spain. Lecture Notes Computer Science, 4477 (2007) 145-152.

[16] K. Shiramita, T. Miyajima, and R. Takiyama. Determination of meat quality by texture analysis. Pattern Recognition Letters, 19 (1998) 1319-1324.

[17] J. Li, J. Tan, F.A. Martz, and H. Heymann. Image texture features as indicators of beef tenderness. Meat Science, 53 (1999) 17-22.

[18] O. Basset, B. Buquet, S. Abouelkaram, P. Delacharte, and J. Culioli. Application of texture analysis for the classification of bovine meat. Food chemistry, 69 (2000) 437-445.

[19] P. Jackman, and D.W. Sun. Recent advances in the use of computer vision technology in the quality assessment of fresh meat. Trends in food science and technology, 22(4) (2011) 185-197.

[20] P. Jackman, and D.W. Sun. Recent advances in image processing using image texture features for food quality assessment. Trends in food science and technology, 29 (2013) 35-43. 
[21] D. Caballero, A. Caro, P.G. Rodríguez, M.L. Durán, M.M. Ávila, R. Palacios, T. Antequera, and T. Pérez-Palacios. Modeling salt diffusion in Iberian ham by applying MRI and data mining. Journal of food engineering, 189 (2016) 115-122.

[22] F. Bajd, M. Skrlep, M. Candek-Potokar, J. Vidmar, and I. Sersa. Use of multiparametric magnetic resonance microscopy for discrimination among different processing protocols and anatomical positions of Slovenian dry-cured hams. Food Chemistry, 197 (2016) 1093-1101.

[23] D.B. Hibbert. Fractals in chemistry. Chemometrics and Intelligent Laboratory Systems, $11(1)(1991) 1-11$.

[24] B.B. Mandelbrot. The fractal geometry of Nature. Ed. W.H. Freeman and Co. New York, New York, U.S.A. 1982.

[25] Y. Tominaga, and L. Fujiwara. Data structure comparison using fractal analysis. Chemometrics and Intelligent Laboratory Systems, 39 (2) (1996) 187-193.

[26] J. Sun, Y.B. Zhang, A.B. Dahl, K. Conradsen, and D. Juul Jensen. Boundary fractal analysis of two cube-oriented grains in partly recrystallized copper. In XVII International Conference on Texture of Materials. ICOTOM 17. Dresden, Germany. 2014.

[27] I. Celigueta-Torres, J.M. Amigo-Rubio, and R. Ipsen. Using fractal image analysis to characterize microstructure of low-fat stirred yogurt manufactured with microparticulated whey protein. Journal of food engineering, 109 (2012) 721-729.

[28] M. Manera, L. Giari, J.A. De Pasquale, and B.S. Dezfuli. Local connected fractal dimmension analysis in gill of fish experimentally exposed to toxicants. Aquatic toxicology, 175 (2016) 12-19. 
[29] S.T. Perisho, D.G. Kelty-Stephen, A. Hajnal, D. Houser, and S.A. Kuczaj II. Fractal scaling in bottlenose dolphin (Tursiops truncates) echolocation: A case study. Physica A, 443 (2016) 221-230.

[30] R. Quevedo, F. Pedreschi, J.M. Bastias, and O. Díaz. Correlation of the fractal enzymatic browning rate with the temperature in mushroom, pear and apple slices. LWT - Food Science and Technology, 65 (2016) 406-413.

[31] R. Quevedo, E. Valencia, G. Cuevas, B. Ronceros, F. Pedreschi, and J.M. Bastías. Color changes in the Surface of fresh cut meat: A fractal kinetic application. Food Research International, 54 (2013) 1430-1436.

[32] P. Zapotoczny, P.M. Szczypinski, and T. Daszkiewicz. Evaluation of the quality of cold meats by computer-assisted image analysis. LWT - Food Science and Technology, 67 (2016) 37-49.

[33] M. Tsuta, J. Sugiyama, and Y. Sagara. Near-infrared Imaging Spectroscopy based on sugar absorption band for melons. Journal of agriculture and food chemistry, 50(1) (2002) 48-52.

[34] G. Polder, G.W.A.M. Van Der Heijden, H. Van Der Hoet, and I.T. Young. Measuring surface distribution of caretones and chlorophyll in ripening tomatoes using imaging spectrometry. Postharvest Biology and Technology, 34 (2004) 117-129.

[35] T. Hastie, R. Tibshirani, and J. Friedman. The Elements of Statistical Learning: Data Mining Inference and Prediction. Ed. Springer-Verlag. New York, New York, U.S.A. 2001. 
[36] U. Fayyad, G. Piatetsky-Shapiro, and P. Smyth. From data mining to knowledge discovery in databases. American Association for Artificial Intelligence, 17 (1996) 37-54.

[37] S. Sayad. Real Time Data Mining. Ed. Self-Help Publishers. Cambridge, Ontario, Canada. 2011.

[38] P. Werbos. Beyond regression: New tools for prediction and analysis in the behavioral science. PhD Thesis, Harvard University. Cambridge, Massachusetts, U.S.A. 1974.

[39] D. Rumelhart, G. Hinton, and R. Williams. Learning internal representations by error propagation. In D. Rumelhart, and J. McClelland. (Eds.) Parallel distributed processing: Explorations in the Microstructures of Cognition, Vol. 1., MIT Press, Cambridge, Massachusetts, U.S.A. 1986. pp. 318-362.

[40] P. Cortez, A. Cerdeira, F. Almeida, T. Matos, and J. Reis. Modeling wine preferences by data mining from physico-chemical properties. Decision Support Systems, 47 (2009) 547-553.

[41] Y.H. Song, S.J., Kim and S.K. Lee. Evaluation of ultrasound for prediction of carcass meat yield and meat quality in Korean Native Cattle (Hanwoo). Asian Australian Journal of Animal Science, 15(4) (2002) 591-595.

[42] P. Cortez, M. Portelinha, S. Rodrigues, V. Cadavez, and A. Teixeira. Lamb meat quality assessment by support vector machines. Neural Processing Letters, 24 (2006) 41-51.

[43] R. Molano, P.G. Rodríguez, A. Caro, and M.L. Durán. Finding the largest area rectangle of arbitrary orientation in a closed contour. Applied mathematics and computation, 218(19) (2012) 9866-9874. 
[44] H. Pan, J. Li, and W. Zhang. Incorporating domain knowledge into medical image clustering. Applied mathematics and computation, 185(2) (2007) 844856.

[45] R.M. Haralick, K. Shanmugam, and I. Dinstein. Textural features for image classification. IEEE Transactions on Man and Cybernetics, 3(6) (1973) 610-621.

[46] R.M. Haralick, and L.G. Shapiro. Computer and Robot Vision. Ed. AddisonWesley. Chicago, Illinois, U.S.A. 1993.

[47] M.M. Galloway. Texture classification using gray level run length. Computer graphics and Image Processing, 4 (1975) 172-179.

[48] L.H. Siew, R.M. Hodgson, and E.J. Wood. Texture measures for carpet wear assessment. IEEE Transactions on Pattern, Analysis and Machine Intelligence, 10(1) (1988) 92-104.

[49] M.M. Ávila, D. Caballero, M.L. Durán, A. Caro, T. Pérez-Palacios, and T. Antequera. Including 3D-textures in a Computer Vision System to analyze quality traits of loin. In X International Conference on Computer Vision Systems (ICVS), Copenhagen, Denmark. Lecture Notes Computer Science, 9163 (2015) 456-465.

[50] M. Sonka, V. Havac, and R. Boyle. Image processing, analysis and machine vision. Ed. International Thomsom Publishing, ITP. Stanford, California, U.S.A. 1999.

[51] C. Sun, and G. Wee. Neighbouring gray level dependence matrix. Computer Vision graphics and image processing, 23 (1982) 341-352. 
[52] D. Caballero, A. Caro, M.M. Ávila, P.G. Rodríguez, T. Antequera, and T. Pérez-Palacios. New fractal features and data mining to determine food quality based on MRI. IEEE Latin America Transactions, 15(9) (2017) 1777-1784.

[53] N. Aggarwal, and R.K. Agrawal. First and second order statistics features for classification of magnetic resonance brain images. Journal of signal and information processing, 3 (2012) 574-580.

[54] S. Peckingpaugh. An improved method for computing gray-level cooccurrence matrix based texture measured. Computer vision, graphics and image processing, 53 (1991) 574-580.

[55] D. Caballero, A. Caro, J.M. Amigo, A.B. Dahl, B.K. Ersbøll, and T. PérezPalacios. Development of a new fractal algorithm to predict quality traits of MRI loins. In XVII International Conference on Computer Analysis of Images and Patterns (CAIP), Ystad, Sweden. Lecture Notes Computer Science, 10424 (2017) 208-218.

[56] Association of Official Analytical Chemist. (AOAC). Official Methods of Analysis of AOAC International (17 th ed.). AOAC International, Gaithersburg, Maryland, U.S.A. 2000.

[57] T. Pérez-Palacios, J. Ruiz, D. Martin, E. Muriel, and T. Antequera. Comparison of different methods for total lipid quantification. Food chemistry, 110 (2008) 1025-1029.

[58] J. Ruiz, C. Garcia, E. Muriel, A.I. Andrés, and J. Ventanas. Influence of sensory characteristics on the acceptability of dry-cured ham. Meat Science, 66 (2002) 11-20. 
[59] I.H. Witten, and E. Frank. Data mining: practical machine learning tools and techniques with Java implementations. Ed. Morgan-Kauffman. San Francisco, California, U.S.A. 2005.

[60] X. Wu, V. Kumar, J. Ross-Quinlan, J. Ghosh, Q. Yang, H. Motoda, G.J. McLachlan, A. Ng, B. Liu, P.S. Yu, Z.H. Zhou, M. Steinbach, D.J. Hand, and D. Steinberg. Top 10 algorithms in data mining. Knowledge and Information Systems, 14 (2008) 1-37.

[61] T. Dietterich. Approximate statistical tests for comparing supervised classification learning algorithms. Neural Computation, 10(7) (1998) 1895-1923.

[62] T. Colton. Statistics in Medicine. Ed. Little Brown and Co. New York, New York, U.S.A. 1974.

[63] R. Hyndman, and A.B. Koehler. Another look at measures of forecast accuracy. International Journal of Forecasting, 22 (2006) 679-688.

[64] D. Caballero, M. Armenteros, M. Asensio, A. Silva, T. Pérez-Palacios, J.E. Llorente, and J. Ventanas. Predicción de la grasa intramuscular en centros de jamón aplicando minería de datos a imágenes de resonancia magnética. In VIII CYTA-CESIA Conference. Badajoz, Spain. 2015.

[65] R. Hyndman. Another look at forecast accuracy metrics for intermittent demand. International Journal of Applied Forecasting, 4 (2006) 43-46.

[66] M.L. Durán, P.G. Rodríguez, J.P. Arias-Nicolas, J. Martin, and C. Disdier. A perceptual similarity method by pairwise comparison in a medical image case. Machine vision and applications, 21 (6) (2010) 865-877.

[67] T. Pérez-Palacios, D. Caballero, A. Caro, and T. Antequera. Low-field Magnetic Resonance Imaging and computational texture features to predict 
moisture and lipid content of loins. In IV Farm Animal Imaging Conference (FAIM). Edinburgh, United Kingdom. 


\section{Figure Captions}

Figure 1. MRIs obtained from different samples of dry-cured loins.

Figure 2. The proposed computational texture algorithm (OPFTA algorithm). A) Input MRI image. B) Largest area rectangle inside of loin contour. C) Calculating ROIs. D) Selecting fractal value. E) Input fractal value in the matrix. F) Calculating features. 
Table 1. Equations of the texture features from the GLRLM algorithm.'

\begin{tabular}{cc}
\hline Features & $=\sum_{i j} P(i, j)^{2}$ \\
ENE & $-\sum_{i j} P(i, j) \log (P(i, j))$ \\
ENT & $=\frac{\sum_{i j}\left(i-\mu_{x}\right)\left(j-\mu_{y}\right) P(i, j)}{\frac{\sigma_{x}}{\sigma_{y}}}$ \\
COR & $=\frac{\sum_{i j}(i, j) P(i, j)-\mu_{x} \mu_{y}}{\sigma_{x} \sigma_{y}}$ \\
HC & $=\sum_{i j} \frac{P(i, j)}{1+(i-j)^{2}}$ \\
IDM & $=\sum_{i j}(i-j)^{2} P(i, j)$ \\
INE & $=\sum_{i j}\left(\left(i-\mu_{x}\right)+\left(j-\mu_{y}\right)\right)^{3} P(i, j)$ \\
CS & $=\sum_{i j}\left(\left(i-\mu_{x}\right)+\left(j-\mu_{y}\right)\right)^{4} P(i, j)$ \\
$\mathrm{CP}$ & $=\sum_{i j}(i-j)^{2}(P(i, j))^{2}$
\end{tabular}

\footnotetext{
${ }^{i} P(i, j)=$ Element with coordinates $(i, j)$ on the grey level co-occurrence matrix. $i=$ Coordinate of the rows.

$\mathrm{j}=$ Coordinate of the columns.

$\mu_{\mathrm{x}}=$ Average value of the rows.

$\mu_{y}=$ Average value of the columns.

$\sigma_{x}=$ Standard Deviation of the rows.

$\sigma_{y}=$ Standard Deviation of the columns.
} 
Table 2. Equations of the texture features from the GLRLM algorithm.'

\begin{aligned} \hline Features & Equation \\ SRE & $=\frac{\sum_{i} \sum_{j}\left(\frac{Q(i, j)}{j^{2}}\right)}{\sum_{i} \sum_{j} Q(i, j)} \\ & =\frac{\sum_{i} \sum_{j} Q(i, j) j^{2}}{\sum_{i} \sum_{j} Q(i, j)} \\$ LRE & $=\frac{\sum_{i}\left(\sum_{j} Q(i, j)\right)^{2}}{\sum_{i} \sum_{j} Q(i, j)} \\$ GLNU & $=\frac{\sum_{j}\left(\sum_{i} Q(i, j)\right)^{2}}{\sum_{i} \sum_{j} Q(i, j)} \\ & =\frac{\sum_{j} \sum_{i} Q(i, j)}{L} \\$ RLNU & $=\frac{\sum_{i} \sum_{j}\left(\frac{Q(i, j)}{i^{2}}\right)}{\sum_{i} \sum_{j} Q(i, j)} \\$ RPC & $=\frac{\sum_{i} \sum_{j} i^{2} Q(i, j)}{\sum_{i} \sum_{j} Q(i, j)} \\$ LGRE & $=\frac{\sum_{i} \sum_{j}\left(\frac{Q(i, j)}{i^{2} j^{2}}\right)}{\sum_{i} \sum_{j} Q(i, j)} \\$ HGRE & $=\frac{\sum_{i} \sum_{j}\left(\frac{i^{2} Q(i, j)}{j^{2}}\right)}{\sum_{i} \sum_{j} Q(i, j)} \\$ SRLGE & $=\frac{\sum_{i} \sum_{j}\left(\frac{j^{2} Q(i, j)}{\sum_{i} \sum_{j} Q(i, j)}\right.}{\sum_{i} \sum_{j} i^{2} j^{2} Q(i, j)} \\ \sum_{i} \sum_{j} Q(i, j) &$ (15) \\ SRHGE & (16) \\ LRLGE & (12) \end{aligned}

${ }^{i} Q(i, j)=$ Element with coordinates $(i, j)$ on the grey level run length matrix.

$\mathrm{i}=$ Coordinate of the rows.

$\mathrm{j}=$ Coordinate of the columns.

$L=$ Total amount of rows in a matrix. 
Table 3. Equations of the texture features from the NGLDM algorithm.'

\begin{tabular}{cl}
\hline Features & Equation \\
SNE & $=\sum_{j} \sum_{i} \frac{R(j, i)}{i^{2}}$ \\
LNE & $=\sum_{j} \sum_{i} i^{2} R(j, i)$ \\
NNU & $=\sum_{i}\left(\sum_{j} R(j, i)\right)^{2}$ \\
SM & $=\sum_{j} \sum_{i}(R(j, i))^{2}$ \\
ENT & $=-\sum_{j} \sum_{i} R(j, i) \log (R(j, i))$
\end{tabular}

\footnotetext{
${ }^{i} R(i, j)=$ Element with coordinates $(i, j)$ on the neichboring grey level dependence matrix. $\mathrm{i}=$ Coordinate of the rows.

$\mathrm{j}=$ Coordinate of the columns.
} 
Table 4. Equations of the texture features from the FTA algorithm.

\begin{tabular}{cc}
\hline Features & $\sum_{i=0}^{n} F_{i}{ }^{2}$ \\
UNI & $\left.\sum_{i=0}^{n} F_{i}^{*} \log _{10}\left(F_{i}\right)\right)$ \\
ENT & $\sum_{i=0}^{n}(i-\mu) * F_{i}$ \\
COR & $\sum_{i=0}^{n} \frac{F_{i}}{1+i^{2}}$ \\
INM & $\sum_{i=0}^{n} F_{i} * i^{2}$
\end{tabular}

${ }^{i} \mathrm{Fi}=$ Value from the vector of local exponent in the position $\mathrm{i}$.

$\mathrm{i}=$ Position in the vector of local exponent.

$\mu=$ Average value of the vector of local exponent. 
Table 5. Equations of the texture features from the OPFTA algorithm.

\begin{tabular}{cc}
\hline Features & Equation \\
\hline UNI & $\sum \sum P(i, j)^{2}$ \\
ENT & $\sum \frac{\sum \sum(i, j) * \log (P(i, j))}{\sum \mu_{x} * \mu_{y} * P(i, j)}$ \\
COR & $\frac{\sigma_{x} / \sigma_{y}}{1+(i-j)^{2}}$ \\
HOM & $\sum \sum P(i, j) *(i-j)^{2}$ \\
INE & $\sum \sum P(i, j)^{2} *(i-j)^{2}$ \\
CON & $\frac{\sigma_{x}}{\mu_{x}}+\frac{\sigma_{y}}{\mu_{y}}$
\end{tabular}

${ }^{i} P(i, j)=$ Element with coordinates $(i, j)$ on the fractal values matrix.

$i=$ Coordinate of the rows.

$\mathrm{j}=$ Coordinate of the columns.

$\mu_{\mathrm{x}}=$ Average value of the rows.

$\mu_{y}=$ Average value of the columns.

$\sigma_{x}=$ Standard Deviation of the rows.

$\sigma_{y}=$ Standard Deviation of the columns. 
Table 6. Computational cost of the most used computer vision algorithms

\begin{tabular}{cccc}
\hline Algorithm & Authors & Reference & $\begin{array}{c}\text { Computational } \\
\text { cost }\end{array}$ \\
\hline GLCM & Haralick et al. & {$[45]$} & $\bigcirc\left(n^{2}\right)$ \\
NGLDM & Sun and Wee & {$[51]$} & $\bigcirc\left(n^{3}\right)$ \\
GLRLM & Galloway & {$[47]$} & $O\left(n^{2}\right)$ \\
GLCM + NGLDM & Durán et al. & {$[66]$} & $\bigcirc\left(n^{3}\right)$ \\
GLRLM & Mandlebrot & {$[24]$} & $\bigcirc\left(n^{3}\right)$ \\
CFA & Caballero et al. & {$[52]$} & $O\left(n^{2} * \log n\right)$ \\
FTA & Caballero et al. & This study & $O\left(n^{2}\right)$ \\
OPFTA & & &
\end{tabular}


Table 7. Correlation coefficient (R) of the prediction equations for physico-chemical parameters of dry-cured loins obtained by applying $M L R^{1}$ on data from different computer vision algorithms at analysing MRI acquired by SE.

\begin{tabular}{|c|c|c|c|c|c|c|}
\hline & GLCM & GLRLM & NGLDM & CFA & FTA & OPFTA \\
\hline MOISTURE (\%) & 0.948 & 0.905 & 0.914 & 0.289 & 0.832 & 0.951 \\
\hline WATER ACTIVITY & 0.950 & 0.906 & 0.911 & 0.303 & 0.828 & 0.954 \\
\hline SALT CONTENT (\%) & 0.949 & 0.908 & 0.907 & 0.340 & 0.795 & 0.956 \\
\hline LIPID CONTENT (\%) & 0.791 & 0.689 & 0.683 & 0.308 & 0.835 & 0.837 \\
\hline COLOR L* & 0.902 & 0.882 & 0.741 & 0.344 & 0.765 & 0.826 \\
\hline COLOR A & 0.851 & 0.737 & 0.788 & 0.201 & 0.765 & 0.854 \\
\hline COLOR B & 0.786 & 0.707 & 0.725 & 0.507 & 0.756 & 0.823 \\
\hline
\end{tabular}

1 MLR: Multiple Linear Regression

SE: Spin Echo

GLCM: Grey level co-occurrence matrix

NGLDM: Neighboring Gray level dependence matrix

GLRLM: Grey level run length matrix

CFA: Classic Fractal Algorithm

FTA: Fractal Texture Algorithm

OPFTA: One Point of Fractal Texture Algorithm 
Table 8. Correlation coefficient ( $R$ ) of the prediction equations for sensory parameters of dry-cured loins obtained by applying MLR ${ }^{1}$ on data from different computer vision algorithms at analysing MRI acquired by SE.

\begin{tabular}{ccccccc}
\hline & GLCM & GLRLM & NGLDM & CFA & FTA & OPFTA \\
\hline REDNESS OF LEAN & 0.853 & 0.605 & 0.762 & 0.308 & 0.838 & $\mathbf{0 . 9 6 7}$ \\
BRIGHTNESS OF LEAN & 0.592 & 0.311 & 0.532 & 0.431 & 0.772 & $\mathbf{0 . 8 4 9}$ \\
MARBLING & 0.812 & 0.563 & 0.746 & 0.272 & 0.829 & $\mathbf{0 . 9 7 4}$ \\
ODOUR INTENSITY & 0.584 & 0.398 & 0.541 & 0.429 & 0.708 & $\mathbf{0 . 8 1 8}$ \\
HARDNESS & 0.770 & 0.524 & 0.693 & 0.231 & 0.838 & $\mathbf{0 . 9 4 7}$ \\
JUICINESS & 0.793 & 0.541 & 0.747 & 0.392 & 0.869 & $\mathbf{0 . 9 7 0}$ \\
SALTY TASTE & 0.585 & 0.170 & 0.430 & 0.420 & 0.701 & $\mathbf{0 . 8 4 4}$ \\
FLAVOR INTENSITY & 0.564 & 0.325 & 0.583 & 0.458 & 0.779 & $\mathbf{0 . 8 8 2}$ \\
FLAVOR PERSISTENCE & 0.461 & 0.255 & 0.452 & 0.455 & 0.705 & $\mathbf{0 . 8 4 8}$ \\
CURED FLAVOR & 0.475 & 0.082 & 0.257 & 0.402 & 0.583 & $\mathbf{0 . 9 0 2}$ \\
RANCID FLAVOR & 0.834 & 0.589 & 0.734 & 0.388 & 0.776 & $\mathbf{0 . 9 0 9}$ \\
\hline
\end{tabular}

1 MLR: Multiple Linear Regression

SE: Spin Echo

GLCM: Grey level co-occurrence matrix

NGLDM: Neighboring Gray level dependence matrix

GLRLM: Grey level run length matrix

CFA: Classic Fractal Algorithm

FTA: Fractal Texture Algorithm

OPFTA: One Point of Fractal Texture Algorithm 
Table 9. Prediction equations for sensory and physico-chemical attributes of dry-cured loins obtained applying MLR' on data obtained using OPFTA at analysing MRI acquired by SE.

\begin{tabular}{|c|c|c|c|}
\hline & PREDICTION EQUATION & MAE & Min - Max \\
\hline MOISTURE $(\%)=$ & $\begin{array}{c}49.568 * \mathrm{UNI}+64.839 * \mathrm{ENT}+4.683 * \mathrm{COR}+16.969 * \mathrm{HOM}+55.767 * \\
\mathrm{INE}-30.923 * \mathrm{CON}+42.805 * \mathrm{EFI}-25.929\end{array}$ & 1.822 & $27.762-37.038$ \\
\hline WATER ACTIVITY = & $\begin{array}{c}0.368 * \mathrm{UNI}+0.334 * \mathrm{ENT}+0.020 * \mathrm{COR}+0.025 * \mathrm{HOM}+0.162 * \mathrm{INE}- \\
0.135 * \mathrm{CON}+0.145 * \mathrm{EFI}+0.566\end{array}$ & 0.014 & $0.840-0.883$ \\
\hline SALT CONTENT (\%) = & $\begin{array}{c}-8.898 * \mathrm{UNI}-7.546 * \mathrm{ENT}-0.506 * \mathrm{COR}-0.265 * \mathrm{HOM}-3.655 * \mathrm{INE}+ \\
3.281 * \mathrm{CON}-3.224 * \mathrm{EFI}+9.312\end{array}$ & 0.289 & $2.534-4.234$ \\
\hline LIPID CONTENT $(\%)=$ & -15.617 * ENT $-7.474 * \mathrm{HOM}-13.201 * \mathrm{INE}-22.415 * \mathrm{EFI}+36.191$ & 1.513 & $9.373-28.321$ \\
\hline COLOR L* = & $\begin{array}{c}14.892 * \mathrm{ENT}+18.902 * \mathrm{HOM}+31.617 * \mathrm{INE}-29.366 * \mathrm{CON}+19.365 * \\
\mathrm{EFI}+26.536\end{array}$ & 1.722 & $32.048-44.175$ \\
\hline COLOR A = & $-2.950 * \mathrm{HOM}-7.324 * \mathrm{INE}+7.488 * \mathrm{CON}-6.859 * \mathrm{EFI}+15.579$ & 1.070 & $12.128-16.400$ \\
\hline COLOR B = & $\begin{array}{c}12.883 * \mathrm{UNI}+3.205 * \mathrm{ENT}-4.705 * \mathrm{HOM}-6.591 * \mathrm{INE}-7.734 * \mathrm{EFI}+ \\
5.644\end{array}$ & 0.757 & $5.942-10.543$ \\
\hline REDNESS OF LEAN = & $\begin{array}{c}4.403 * \mathrm{ENT}-1.131 * \mathrm{COR}+0.826 * \mathrm{HOM}-1.253 * \mathrm{INE}+1.711 * \mathrm{CON}+ \\
5.137 * \mathrm{EFI}+3.062\end{array}$ & 0.740 & $6.410-9.026$ \\
\hline BRIGHTNESS OF LEAN = & $2.616 * \mathrm{UNI}-0.754 * \mathrm{INE}-6.529 * \mathrm{EFI}+4.764$ & 0.553 & $3.742-5.689$ \\
\hline MARBLING = & $\begin{array}{c}3.262 * \mathrm{UNI}-8.596 * \mathrm{ENT}+2.673 * \mathrm{COR}-1.556 * \mathrm{HOM}+1.797 * \mathrm{INE}- \\
5.698 * \mathrm{CON}-11.163 * \mathrm{EFI}+14.636\end{array}$ & 0.254 & $2.263-8.276$ \\
\hline ODOUR INTENSITY = & $\begin{array}{c}-1.657 * \mathrm{ENT}+0.689 * \mathrm{COR}+0.703 * \mathrm{HOM}-0.596 * \mathrm{INE}+0.792 * \mathrm{CON} \\
-7.707 * \mathrm{EFI}+8.223\end{array}$ & 0.676 & $5.563-7.842$ \\
\hline HARDNESS = & $\begin{array}{c}-2.218 * \mathrm{UNI}+3.948 * \mathrm{ENT}-1.231 * \mathrm{COR}+1.130 * \mathrm{HOM}-1.128 * \mathrm{INE}+ \\
3.647 * \mathrm{CON}+3.244 * \mathrm{EFI}+1.675\end{array}$ & 0.897 & $4.273-7.411$ \\
\hline JUICINESS = & $\begin{array}{c}1.008 * \mathrm{UNI}-3.229 * \mathrm{ENT}+1.176 * \mathrm{COR}-1.511 * \mathrm{CON}-5.796 * \mathrm{EFI}+ \\
8.809\end{array}$ & 0.765 & $3.900-6.700$ \\
\hline SALTY TASTE $=$ & $4.354 * \mathrm{UNI}+2.113 * \mathrm{ENT}-1.231 * \mathrm{INE}-5.437 * \mathrm{EFI}+3.312$ & 0.494 & $4.194-5.979$ \\
\hline
\end{tabular}

1 MLR: Multiple Linear Regression

SE: Spin Echo 
FLAVOR INTENSITY = FLAVOR PERSISTENCE = CURED FLAVOR = RANCID FLAVOR =
$1.513 * \mathrm{UNI}-5.347 * \mathrm{EFI}+6.761$

$0.844 * \mathrm{UNI}+0.630 * \mathrm{CON}-5.164 * \mathrm{EFI}+5.698$

$1.143 * \mathrm{HOM}-0.501 * \mathrm{INE}-4.126 * \mathrm{EFI}+6.135$

$0.487 * \mathrm{UNI}+1.506 * \mathrm{ENT}-0.320 * \mathrm{COR}+0.386 * \mathrm{HOM}-0.595 * \mathrm{INE}+$

$2.156 * \mathrm{EFI}+0.477$
0.418

0.421

0.399

0.260
$6.060-7.288$

$4.882-6.365$

$5.524-7.137$

$1.359-2.516$ 
Figure 1.
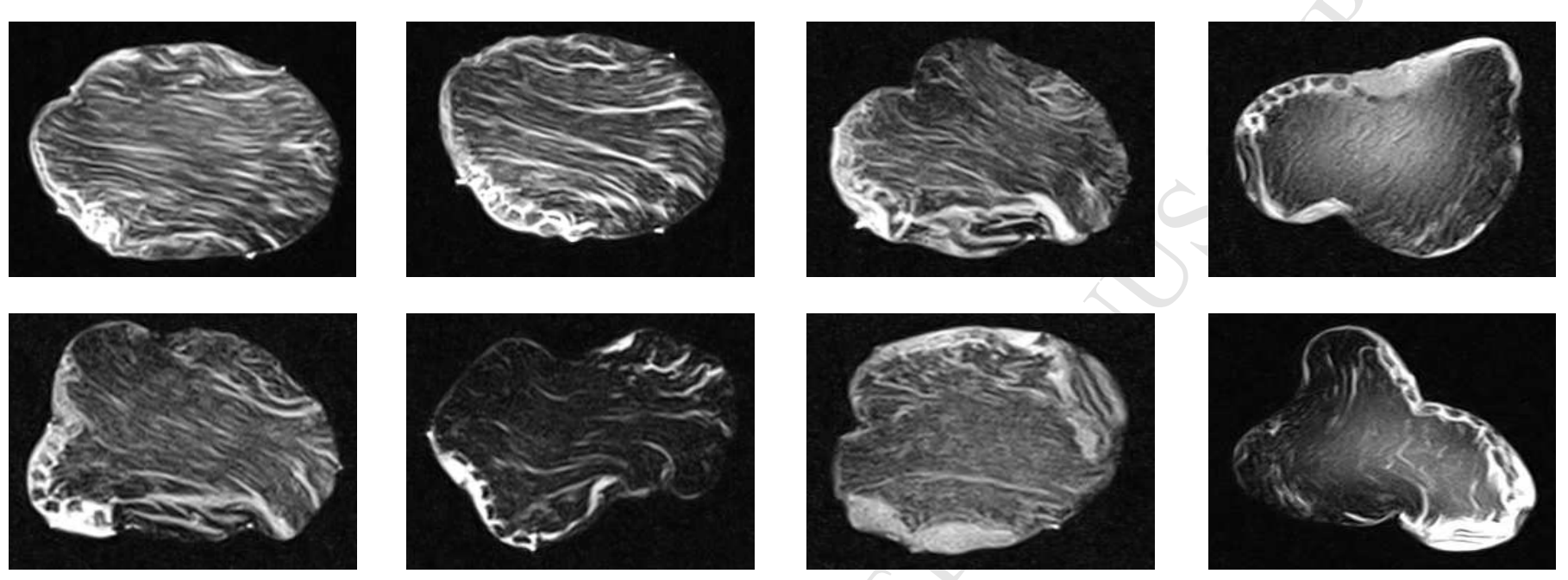
Figure 2.

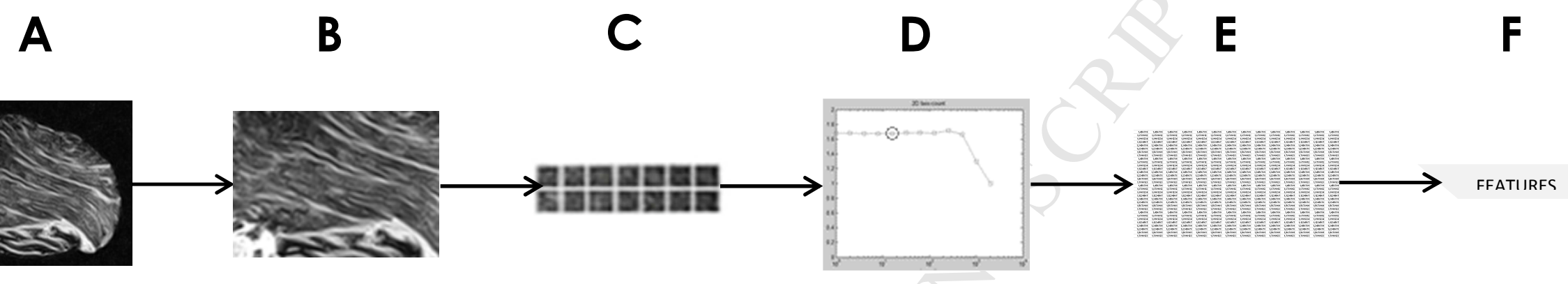




\section{HIGHLIGHTS}

A new fractal properties algorithm was developed and tested in this study.

Different image analysis algorithms were tested to study some parameters of loin.

A new fractal properties algorithm shows lower computational costs than other algorithms.

The proposed algorithm join to MRI could be a tool for the meat industries. 